\title{
Concerns of the application of lightning protection risk assesment for small structures
}

\begin{abstract}
International Standards on lightning protection risk assessment (IEC 62305-2: 2010) are well accepted and applied in many parts of the world. During the last few years a large number of lightning related accidents, especially due to direct strikes, have been reported from Asia and Africa where the lightning struck objects are small structures. Information gathered from several investigations done on these cases have been used to determine the level of protection needed for these structures as per the standard risk assessment. In many cases the outcome was the none-requirement of any LPS for such structures or a risk factor for direct strike probability which is less than the critical value. As there are millions of similar structures in these regions, we propose the development of a separate risk assessment algorithm for such structures, especially in underdeveloped countries.
\end{abstract}

Keyword: IEC; Lightning injuries; Risk assesment; Small strctures 